\title{
DIFFEOMORPHISMS ADMITTING SRB MEASURES AND THEIR REGULARITY
}

\author{
Jin НАТомото
}

\begin{abstract}
We are interested in the stochastic property of some "Anosov-like" system. In this paper we will treat a transitive and partially hyperbolic diffeomorphism $f$ of a 2dimensional torus with uniformly contracting direction, and show that if $f$ is of $C^{2}$ and admits an SRB measure, then $f$ is an Anosov diffeomorphism. In our proof we use the Pujals-Sambarino theorem for $C^{2}$ diffeomorphisms with dominated splitting. In the case of $C^{1+\alpha}$ the above statement is not true in general, i.e. we can construct a $C^{1+\alpha}$ counter example of Maneville-Pomeau type.
\end{abstract}

\section{Introduction}

We know that if $f$ is a $C^{2}$-Anosov diffeomorphism of a compact manifold $M$, then $f$ admits an Sinai-Ruelle-Bowen measure $\mu$ (or SRB measure), i.e., $\mu$ has absolutely continuous conditional measures on unstable manifolds (Sinai [23]). This measure $\mu$ is isomorphic to a Berunulli shift, and it has exponential decay of correlations for Hölder continuous functions, and furthermore satisfies that

$$
\lim _{n \rightarrow \infty} \frac{1}{n} \sum_{j=0}^{n-1} \varphi\left(f^{j} x\right)=\int \varphi d \mu
$$

for any continuous function $\varphi: M \rightarrow \mathbf{R}$ and for Lebesgue almost every $x \in M$. This result has been extended to Axiom A attractors by Bowen and Ruelle (e.g. [4]).

Let $f$ be a $C^{1+\alpha}$-diffeomorphism of a 2-dimensional torus $\mathbf{T}^{2}(0<\alpha \leq 1)$ and $\Gamma$ be an $f$-invariant set of $\mathbf{T}^{2}$, i.e. $f(\Gamma)=\Gamma$. We say that $f$ is partially hyperbolic with contracting direction on $\Gamma$ if there exist a norm $\|\cdot\|$ on $\mathbf{T}^{2}$ and $0<\lambda_{1}<\lambda_{2}$ with $\lambda_{1}<1$ so that each $x \in \mathbf{T}^{2}$ has a $D_{x} f$-invariant decomposition $\mathrm{T}_{x} \mathbf{T}^{2}=E_{1}(x) \oplus E_{2}(x)$ of subspaces $E_{1}(x)$ and $E_{2}(x)$ such that

1991 Mathematics Subject Classification. Primary 37C40, 37D20.

Key words and phrases. Anosov diffeomophism, SRB measure, first return map.

Received October 7, 2005; revised November 21, 2005. 


$$
\left\|\left.D_{x} f\right|_{E_{1}(x)}\right\| \leq \lambda_{1}, \quad\left\|\left.D_{x} f\right|_{E_{2}(x)}\right\| \geq \lambda_{2}
$$

(Here $D_{x} f$ denotes the derivative of $f$ at $x$ ). Moreover, when $\lambda_{2}>1, f$ is called hyperbolic on $\Gamma$ (or $\Gamma$ is a hyperbolic set for $f$ ). We call that $f$ is an Anosov diffeomorphism if $f$ is hyperbolic on the entire space $\mathbf{T}^{2} . f$ is said to be topologically transitive if there exists a point $x \in \mathbf{T}^{2}$ such that its orbit $\left\{f^{n}(x)\right\}_{n \in \mathbf{Z}}$ is dense in $\mathbf{T}^{2}$.

The purpose of this paper is to show the following two theorems:

THeOrem A. Let $f$ be a $C^{2}$-diffeomorphism of $\mathbf{T}^{2}$. Then $f$ is an Anosov diffeomorphism if and only if the following holds:

(1) $f$ is partially hyperbolic with contracting direction on $\mathbf{T}^{2}$,

(2) $f$ is topologically transitive and

(3) $f$ admits an SRB measure.

Theorem $\mathrm{A}$ is false in the case when $f$ is of $C^{1+\alpha}(0<\alpha<1)$, i.e.

THEOREM B. For $\alpha \in(0,1)$ there exists a $C^{1+\alpha}$-diffeomorphism $f$ such that

(1) $f$ is partially hyperbolic with contracting direction on $\mathbf{T}^{2}$ but not an Anosov diffeomorphism,

(2) $f$ is topologically transitive and

(3) $f$ admits an SRB measure.

\section{Definitions and preliminaries}

Fix $\alpha \in(0,1]$ and let $f$ be a $C^{1+\alpha}$-diffeomorphism of $\mathbf{T}^{2}$. Assume that $f$ is partially hyperbolic and has contracting direction. Then $f$ has the decomposition satisfying (1.1). Let $\mu$ be an $f$-invariant probability measure on $\mathbf{T}^{2}$. By Birkhoff's ergodic theorem there exist a set $Y_{\mu}$ with full $\mu$-measure and real numbers $\chi_{1}(x)<\chi_{2}(x)\left(x \in Y_{\mu}\right)$ which satisfy the following:

$$
\lim _{n \rightarrow \pm \infty} \frac{1}{n} \log \left\|\left.D_{x} f^{n}\right|_{E_{i}(x)}\right\|=\chi_{i}(x) \quad(i=1,2) .
$$

We call $\chi_{i}(x)(i=1,2)$ the Lyapunov exponents of $\mu$ at $x \in Y_{\mu}$. By (1.1) we have

$$
\chi_{1}(x) \leq \log \lambda_{1}<\log \lambda_{2} \leq \chi_{2}(x) \quad\left(x \in Y_{\mu}\right) .
$$

We say that $\mu$ is an $S R B$ measure if (i) $\chi_{2}(x)>0$ and (ii) $\mu$ has the conditional measures which are absolutely continuous w.r.t. the Lebesgue measures on unstable manifolds, which is defined as follows:

If $\chi_{2}(x)>0$ for any $x \in Y_{\mu}$, then it is well known (see [16]) that there exists $\varepsilon_{0}>0$ sufficiently small and the local unstable manifold $W_{l o c}^{u}(x)$ such that

$$
f^{-1}\left(W_{l o c}^{u}(x)\right) \subset W_{l o c}^{u}\left(f^{-1}(x)\right),
$$

for any $y \in W_{l o c}^{u}(x)$ and $n \geq 0$ 


$$
d^{u}\left(f^{-n}(x), f^{-n}(y)\right) \leq C(x) \exp \left(\left(-\chi_{2}(x)+\varepsilon_{0}\right) n\right) d^{u}(x, y)
$$

and $E_{2}(x)=T_{x} W_{l o c}^{u}(x)$, where $d^{u}$ denotes the Riemannian metric on $W_{l o c}^{u}(x)$ and $C(x)$ satisfies

$$
\lim _{n \rightarrow \pm \infty} \frac{1}{n} \log C\left(f^{n}(x)\right)=0 .
$$

The unstable manifold $W^{u}(x)$ is defined by

$$
W^{u}(x)=\bigcup_{n \geq 0} f^{n}\left(W_{l o c}^{u}\left(f^{-n}(x)\right)\right) .
$$

Since $\chi_{1}(x)<0$ for any $x \in Y_{\mu}$, the local stable manifold $W_{\text {loc }}^{s}(x)$ exists ([16]) and satisfies

$$
f\left(W_{l o c}^{s}(x)\right) \subset W_{l o c}^{s}(f(x)),
$$

for any $y \in W_{l o c}^{s}(x)$ and $n \geq 0$

$$
d^{s}\left(f^{n}(x), f^{n}(y)\right) \leq D(x) \exp \left(\left(\chi_{1}(x)+\varepsilon_{0}\right) n\right) d^{s}(x, y)
$$

and $E_{1}(x)=T_{x} W_{l o c}^{s}(x)$, where $d^{s}$ denotes the Riemannian metric on $W_{l o c}^{s}(x)$ and $D(x)$ satisfies $\lim _{n \rightarrow \pm \infty}(1 / n) \log D\left(f^{n}(x)\right)=0$. The stable manifold $W^{s}(x)$ is also defined.

Let $\mathscr{B}$ denote the Borel $\sigma$ algebra of $\mathbf{T}^{2}$. For any measurable partition $\xi$ of $\mathbf{T}^{2}$ we denote by $\mathscr{B} \xi$ the set of all Borel subsets which consist of the unions of the elements of $\xi$. A measurable partition $\xi$ of $\mathbf{T}^{2}$ defines a family of measures $\left\{\mu_{x}^{\xi}\right\}$ $(\mu$-a.e. $x)$ such that for $\mu$-a.e. $x$ and $B \in \mathscr{B}, \mu_{x}^{\xi}(B)$ is a $\mathscr{B}_{\xi}$-measurable function of $x$ and

$$
\mu(E \cap B)=\int_{E} \mu_{x}^{\xi}(B) d \mu(x) \quad\left(E \in \mathscr{B}_{\xi}\right) .
$$

If there exists a sequence $\left\{\xi_{i}\right\}_{i \geq 1}$ of countable measurable partitions such that

$$
\xi_{1} \leq \xi_{2} \leq \cdots \leq \bigvee_{i \geq 1} \xi_{i}=\xi
$$

then $\mu_{x}^{\xi}(\xi(x))=1$ where $\xi(x)$ denotes an element of $\xi$ containing $x$. The family of measures $\left\{\mu_{x}^{\xi}\right\}$ ( $\mu$-a.e. $x$ ) is said to be the canonical system of conditional measures of $\mu$ w.r.t. $\xi$ (see [20]).

We assume that a measurable partition $\xi^{u}$ of $\mathbf{T}^{2}$ is subordinate to the $W^{u}$-foliation, i.e., $\xi^{u}$ satisfies that $(1) \xi^{u}(x) \subset W^{u}(x)$ and $(2) \xi^{u}(x)$ contains an open set in $W^{u}(x)$ for $\mu$-a.e.x. Let $\left\{\mu_{x}^{u}\right\} \quad(\mu$-a.e. $x)$ denote a canonical system of conditional measures of $\mu$ w.r.t. $\xi^{u}$ and $m_{x}^{u}$ denote the Lebesgue measure on $W^{u}(x)$. If $\mu_{x}^{u}$ is absolutely continuous w.r.t. $m_{x}^{u}$ for $\mu$-a.e. $x$ $\left(\mu_{x}^{u} \ll m_{x}^{u}\right)$, then we say that $\mu$ has the conditional measures which are absolutely continuous w.r.t. the Lebesgue measures on unstable manifolds (see [13]). If $\mu$ is an SRB measure, then so does every element in the ergodic 
decomposition of $\mu$ for a set of $\mu$-full measure. If $\mu_{x}^{u} \ll m_{x}^{u}(\mu$-a.e. $x)$, then we know that $\left.\mu_{x}^{u} \sim m_{x}^{u}\right|_{\xi^{u}(x)}$ (see [13], [14], [7]).

\section{Proof of Theorem A}

Let $f$ be a $C^{2}$-partially hyperbolic diffeomorphism with contracting direction on $\mathbf{T}^{2}$ and $\mu$ be an $f$-invariant probability measure on $\mathbf{T}^{2}$. Let us denote $I_{\varepsilon}=[-\varepsilon, \varepsilon]$ for any $0<\varepsilon \leq 1$ and $\operatorname{Emb}^{2}\left(I_{1}, \mathbf{T}^{2}\right)$ as the set of $C^{2}$-embeddings of $I_{1}$ into $\mathbf{T}^{2}$ equipped with the $C^{2}$-metric. We can find (see [9]) two continuous maps $\phi^{s}: \mathbf{T}^{2} \rightarrow \operatorname{Emb}^{2}\left(I_{1}, \mathbf{T}^{2}\right)$ and $\phi^{c u}: \mathbf{T}^{2} \rightarrow \operatorname{Emb}^{2}\left(I_{1}, \mathbf{T}^{2}\right)$ such that for any $0<\varepsilon \leq 1$ the local stable and center unstable manifolds $\tilde{W}_{\varepsilon}^{s}(x)=\phi^{s}(x)\left(I_{\varepsilon}\right)$ and $\tilde{W}_{\varepsilon}^{c u}(x)=$ $\phi^{c u}(x)\left(I_{\varepsilon}\right)$ satisfies the following:

(i) $T_{x} \tilde{W}_{\varepsilon}^{s}(x)=E_{1}(x)$ and $T_{x} \tilde{W}_{\varepsilon}^{c u}(x)=E_{2}(x)$,

(ii) for any $\varepsilon_{1} \in(0,1), f\left(\tilde{W}_{\varepsilon_{1}}^{s}(x)\right) \subset \tilde{W}_{\varepsilon_{1}}^{s}(f(x))$,

(iii) for any $\varepsilon_{1} \in(0,1)$, there exists $\varepsilon_{2} \in(0,1)$ such that $f^{-1}\left(\tilde{W}_{\varepsilon_{2}}^{c u}(x)\right) \subset$ $\tilde{W}_{\varepsilon_{1}}^{c u}\left(f^{-1}(x)\right)$.

Thus there exists $\delta>0$ such that if $d(x, y)<\delta$ ( $d$ denotes the Riemannian metric on $\mathbf{T}^{2}$ ), then $\tilde{W}_{\varepsilon}^{s}(x)$ and $\tilde{W}_{\varepsilon}^{c u}(y)$ have a single transverse intersection point, so we write

$$
[x, y]=\tilde{W}_{\varepsilon}^{s}(x) \cap \tilde{W}_{\varepsilon}^{c u}(y) \quad\left(x, y \in \mathbf{T}^{2} \text { with } d(x, y)<\delta\right) .
$$

We denote by $\tilde{d}^{s}$ and $\tilde{d}^{c u}$ the Riemannian metric on $\tilde{W}_{\varepsilon}^{s}(x)$ and $\tilde{W}_{\varepsilon}^{c u}(x)$ respectively. Let $B(x, r)$ be the ball centered at $x$ with radius $r$. Since $\varepsilon_{0}>0$ is small enough, without loss of generality we can assume that the diameater of $\tilde{W}_{\varepsilon}^{c u}(x)$ (respectively $\left.\tilde{W}_{\varepsilon}^{s}(x)\right)$ is greater than $W_{l o c}^{u}(x)$ (respectively $W_{l o c}^{s}(x)$ ) for $x \in Y_{\mu}$.

Lemma 3.1. For any $x \in Y_{\mu}, W_{l o c}^{u}(x)$ is contained in $\tilde{W}_{\varepsilon}^{c u}(x)$ and $W_{l o c}^{s}(x)$ is contained in $\tilde{W}_{\varepsilon}^{s}(x)$.

Proof. Let $C(x)$ be as in (2.1) and $\delta$ be as in (3.1). Firstly we prove that $W_{l o c}^{u}(x) \cap B\left(x, \delta C(x)^{-1}\right) \subset \tilde{W}_{\varepsilon}^{c u}(x)$ for $x \in Y_{\mu}$. To do so, assume that there exist $x \in Y_{\mu}$ and $y \in W_{l o c}^{u}(x) \cap B\left(x, \delta C(x)^{-1}\right) \backslash \tilde{W}_{\varepsilon}^{c u}(x)$. By (2.1) we have $d^{u}\left(f^{-n}(y), f^{-n}(x)\right)<\delta$ for $n \geq 1$ and then define

$$
\left[f^{-n}(y), f^{-n}(x)\right]=\tilde{W}_{\varepsilon}^{s}\left(f^{-n}(y)\right) \cap \tilde{W}_{\varepsilon}^{c u}\left(f^{-n}(x)\right) \quad(n \geq 1) .
$$

Since $y \neq x$ and $\left[f^{-n}(y), f^{-n}(x)\right]=f^{-n}[y, x]$, we have

$$
f^{-n}([y, x]) \in \tilde{W}_{\varepsilon}^{s}\left(f^{-n}(y)\right), \quad f^{-n}([y, x]) \neq f^{-n}(y) \quad(n \geq 1) .
$$

Since $f$ is uniformly contracting along $E_{1}$, we have $\left\|\left.D_{z} f^{-n}\right|_{E_{1}(z)}\right\| \geq \lambda_{1}^{-n}$ $\left(z \in \tilde{W}_{\varepsilon}^{s}(y)\right)$ and then

$$
\varepsilon>\tilde{d}^{s}\left(f^{-n}([y, x]), f^{-n}(y)\right) \geq \lambda_{1}^{-n} \tilde{d}^{s}([y, x], y) \quad(n \geq 1) .
$$

This is a contradiction. 
(2.1) and (2.2) ensure the existence of $i \geq 1$ such that

$$
f^{-i}\left(W_{l o c}^{u}(x)\right) \subset W_{l o c}^{u}\left(f^{-i}(x)\right) \cap B\left(f^{-i}(x), \delta C\left(f^{-i}(x)\right)^{-1}\right) \subset \tilde{W}_{\varepsilon}^{c u}\left(f^{-i}(x)\right)
$$

for any $x \in Y_{\mu}$. Thus we have that $W_{l o c}^{u}(x) \cap B(x, \varepsilon) \subset \tilde{W}_{\varepsilon}^{c u}(x)$ for any $x \in Y_{\mu}$.

Next we use the similar argument to prove the last part of the lemma. Assume that $W_{l o c}^{s}(x) \cap B\left(x, \delta D(x)^{-1}\right) \not \subset \tilde{W}_{\varepsilon}^{s}(x)$ for some $x \in Y_{\mu}$. Here $D(x)$ be as in (2.3). Then there exists $y \in W_{l o c}^{s}(x) \cap B\left(x, \delta D(x)^{-1}\right) \backslash \tilde{W}_{\varepsilon}^{s}(x)$. From this, we have $d^{s}\left(f^{n}(y), f^{n}(x)\right)<\delta$ for $n \geq 1$ and define

$$
\left[f^{n}(y), f^{n}(x)\right]=\tilde{W}_{\varepsilon}^{s}\left(f^{n}(y)\right) \cap \tilde{W}_{\varepsilon}^{c u}\left(f^{n}(x)\right) \quad(n \geq 1) .
$$

Notice that $[y, x] \neq x$ because of $\tilde{W}_{\varepsilon}^{s}(y) \cap \tilde{W}_{\varepsilon}^{s}(x)=\emptyset$, and (2.3) shows that

$$
\begin{aligned}
\tilde{d}^{u}\left(f^{n}([y, x]), f^{n}(x)\right) \leq & \tilde{d}^{s}\left(f^{n}([y, x]), f^{n}(y)\right)+d^{s}\left(f^{n}(y), f^{n}(x)\right) \\
\leq & \lambda_{1}^{n} \tilde{d}^{s}([y, x], y)+D(x) \exp \left(\left(\chi_{1}(x)+\varepsilon_{0}\right) n\right) d^{s}(x, y) \\
& (n \geq 1) \\
\rightarrow & 0 \quad(n \rightarrow \infty) .
\end{aligned}
$$

By the first statement of the lemma, $\tilde{W}_{\varepsilon}^{c u}\left(f^{n}(x)\right) \supset W_{l o c}^{u}\left(f^{n}(x)\right) \cap B\left(f^{n}(x), \varepsilon\right)$ and, since $f^{n}\left(W_{l o c}^{u}(x) \cap B(x, \varepsilon)\right)$ is expanding along $E_{2}$, it doesn't happen that $\tilde{d}^{u}\left(f^{n}([y, x]), f^{n}(x)\right) \rightarrow 0(n \rightarrow \infty)$. This contradicts (3.2). So we have $W_{l o c}^{s}(x) \cap B\left(x, \delta D(x)^{-1}\right) \subset \tilde{W}_{\varepsilon}^{s}(x)$ for any $x \in Y_{\mu}$. Then as in the proof of the first statement, we have that $W_{l o c}^{s}(x) \cap B(x, \varepsilon) \subset \tilde{W}_{\varepsilon}^{s}(x)$ for any $x \in Y_{\mu}$.

We say that $I \subset \tilde{W}_{\varepsilon}^{c u}(x)$ is an interval if there exist $y, z \in I_{\varepsilon}(y<z)$ such that $I=\phi^{c u}(x)([y, z])$. For $0<\varepsilon^{\prime}<\varepsilon$ we identify $\tilde{W}_{\varepsilon^{\prime}}^{c u}(x)$ with $I_{\varepsilon^{\prime}} \subset \mathbf{R}$ if there is no confusion. For any fixed point $p$, without loss of generality we can assume that all the eigenvalues of $D_{p} f$ are positive (by replacing $f$ by $f^{2}$ if necessary).

Lemma 3.2 ([11] Lemma 4.1). Let $p \in \mathbf{T}^{2}$ be a fixed point satisfying

$$
\left\|\left.D_{p} f\right|_{E_{2}(p)}\right\|=1, \quad f^{-1}\left(\tilde{W}_{\varepsilon^{\prime}}^{c u}(p)\right) \subset \tilde{W}_{\varepsilon^{\prime}}^{c u}(p)
$$

for some $0<\varepsilon^{\prime}<\varepsilon$. Then, for any interval $J \subset \tilde{W}_{\varepsilon^{\prime}}^{c u}(p)$ containing $p$,

$$
\sum_{i=0}^{\infty} \ell\left(f^{-i}(J)\right)=\infty .
$$

Here $\ell(I)$ denotes the length of $I$.

By $\left\|\left.D_{p} f\right|_{E_{1}(x)}\right\|<\lambda_{1}$ for any $x \in \mathbf{T}^{2}$, the following statement is a result of Pujals-Sambarino $([18])$.

Lemma 3.3 ([18] Corollary 3.5). Assume that $p \in \mathbf{T}^{2}$ is a fixed point such that

$$
f^{-1}\left(\tilde{W}_{\varepsilon^{\prime}}^{c u}(p)\right) \subset \tilde{W}_{\varepsilon^{\prime}}^{c u}(p)
$$


for some $0<\varepsilon^{\prime}<\varepsilon$ and fix an interval $J \subset \tilde{W}_{\varepsilon^{\prime}}^{c u}(p)$. Then there exists $L=$ $L(J, \varepsilon)>0$ such that

$$
L^{-1} \ell\left(f^{-n}(J)\right) \leq \ell\left(\left[f^{-n}(J), q\right]\right) \leq L \ell\left(f^{-n}(J)\right)
$$

for any $q \in \tilde{W}_{\varepsilon^{\prime}}^{s}(p)$ and $n \geq 0$.

Remark 3.4. If $f$ is topologically transitive, then any fixed point of $f$ satisfies the condition of Lemma 3.3.

Let $\Gamma$ be an $f$-invariant compact set. We say that $f$ has a dominated splitting on $\Gamma$ if there exist $C>0$ and $0<\lambda<1$ such that each $x \in \Gamma$ is decomposed $\mathrm{T}_{x} \mathbf{T}^{2}=E_{1}(x) \oplus E_{2}(x)$ into the sum of $D_{x} f$-invariant subspaces $E_{1}(x)$ and $E_{2}(x)$ which satisfies

$$
\left\|\left.D_{x} f^{n}\right|_{E_{1}(x)}\right\|\left\|\left.D_{f^{n}(x)} f^{-n}\right|_{E_{2}\left(f^{n}(x)\right)}\right\| \leq C \lambda^{n} \quad(n \geq 0) .
$$

Clearly, if $f$ is partially hyperbolic with contracting direction on $\Gamma$, then $f$ has a dominated splitting on $\Gamma$.

We denote by $\Omega(f)$ the set of points $x \in \mathbf{T}^{2}$ such that for any neighborhood $V$ of $x$ there exists $n>0$ satisfying $f^{n}(V) \cap V \neq \emptyset$. We say that an $n$-periodic point $p$ is hyperbolic if the absolute values of eigenvalues of $D_{p} f^{n}$ are different from 1 and is sink (respectively source) if all the absolute values of eigenvalues of $D_{p} f^{n}$ are smaller(respectively larger) than 1 . If $\mu$ has positive and negative Lyapunov exponents then the set of hyperbolic periodic points is not empty ([12]).

Lemma 3.5 ([18]). Assume that $f$ has a dominated splitting on $\Omega(f)$ and all the periodic points in $\Omega(f)$ are hyperbolic. Then $\Omega(f)$ is represented as a union $\Omega(f)=\Gamma_{1} \cup \Gamma_{2}$ of $\Gamma_{1}$ and $\Gamma_{2}$ where $\Gamma_{1}$ is a hyperbolic set for $f$ and $\Gamma_{2}$ consists of a finite union of periodic simple closed curves $\mathscr{C}_{1}, \ldots, \mathscr{C}_{n}$ such that each $\mathscr{C}_{i}$ is normally hyperbolic and $f^{m_{i}}: \mathscr{C}_{i} \rightarrow \mathscr{C}_{i}$ is conjugated to an irrational rotation $\left(m_{i}\right.$ denotes the period of $\mathscr{C}_{i}$ ).

In particular, in the case when $\Omega(f)=\mathbf{T}^{2}, f$ is an Anosov diffeomorphism.

Remark 3.6. If $f$ is topologically transitive, then all the periodic points are not sink nor source.

Proof of Theorem A. Assume that $f$ is an Anosov diffeomorphism. Then $f$ satisfies the conditions (1)-(3) of Theorem A. Indeed, the condition (1) is obtained from the definitions of Anosov and partially hyperbolic diffeomorphisms. Since any Anosov diffeomorphism of $\mathbf{T}^{2}$ is topologically conjugate to some hyperbolic toral automorphism ([6] Theorem 6.3) and hyperbolic toral automorphisms are topologically transitive, we have the condition (2). The condition (3) is the direct consequence of [23] as stated in Introduction. Therefore it remains only to show that the converse statement holds.

To do so we prepare the following claim. 
Claim. All the periodic points of $\mathbf{T}^{2}$ are hyperbolic.

If we have the claim, then $f$ satisfies the assumption of Lemma 3.5. Thus we have the conclusion.

To show the claim, we choose an arbitrary periodic point $p$ with period $n$. To simplicity we replace $f$ by $f^{n}$. Then $p$ is a fixed point of $f$. Since $f$ is uniformly contracting along $E_{1}$, we have to show that $\left\|\left.D_{p} f\right|_{E_{2}(p)}\right\|>1$. Since $f$ is topologically transitive, by Remark 3.6 it doesn't happen that $\left\|\left.D_{p} f\right|_{E_{2}(p)}\right\|<1$. Assume that $\left\|\left.D_{p} f\right|_{E_{2}(p)}\right\|=1$. Then we lead a contradiction by using the method in the proof of Theorem $\mathrm{A}$ in [11].

Fix $0<\varepsilon_{1}<\min \left\{\varepsilon, \delta /\left(2 \lambda_{1}\right)\right\}$ and define a neighborhood $\mathscr{P}$ of $p$ by

$$
\mathscr{P}=\left\{[y, x] \mid y \in \tilde{W}_{\varepsilon_{1}}^{c u}(p), x \in \tilde{W}_{\varepsilon_{1}}^{s}(p)\right\} .
$$

When we identify $\tilde{W}_{\varepsilon_{1}}^{c u}(p)$ with an interval $I_{\varepsilon_{1}} \subset \mathbf{R}$ and $p$ is a fixed point of $f$, the graph of $\left.f\right|_{\tilde{W}_{\varepsilon_{1}}^{c u}(p)}$ satisfies $|f|_{\tilde{W}_{\varepsilon_{1}}^{c u}(p)}(y)|>| y \mid$ for any $y \in \tilde{W}_{\varepsilon_{1}}^{c u}(p) \backslash\{p\}$ (Figure 1) because $f$ is topologically transitive. Since $f^{-1}$ is uniformly expanding along $E_{1}, f^{-1}(\mathscr{P})$ intersects $\mathscr{P}$ transeversely along stable direction (see Figure 2 ).

Let $\xi^{u}$ be a measurable partition subordinate to the $W^{u}$-foliation and $\left\{\mu_{x}^{u}\right\}$ $(\mu$-a.e. $x)$ denote a canonical system of conditional measures of $\mu$ w.r.t. $\xi^{u}$. Since $\mu$ is an SRB measure, we can take a measurable function $g: \mathbf{T}^{2} \rightarrow \mathbf{R}$ satisfying

$$
g(z)=\frac{d \mu_{y}^{u}}{d m_{y}^{u}}(z)
$$

for $\mu$-a.e.y and $m_{y}^{u}$-a.e. $z \in \xi^{u}(y)([13])$. Here $m_{y}^{u}$ denotes the Lebesgue measure on $W^{u}(y)$. Moreover it is known ([14] Corollary 6.1.4) that for $\mu$-a.e. $y, g$ is strictly positive on $\xi^{u}(y)$ and $\log g$ is Lipshitz continuous on $\xi^{u}(y)$.

By the definition of $\xi^{u}$, there exist $r>0, x_{0} \in Y_{\mu}$ and a closed set $A \subset Y_{\mu} \cap B\left(x_{0}, r\right)$ with $\mu(A)>0$ such that for any $y \in A$

(a) $\xi^{u}(y) \supset B^{u}(y, 2 r)$ where $B^{u}(y, 2 r)$ denotes the ball centered at $y$ with radius $2 r$ in $W^{u}(y)$,

(b) $\lim _{n \rightarrow \pm \infty} \frac{1}{n} \log \left\|\left.D_{z} f^{n}\right|_{E_{2}(z)}\right\|=\chi_{2}(z)>0$ for $m_{y}^{u}$-a.e. $z \in \xi^{u}(y)$,

(c) there exists $C_{0}>0$ (independent of $y$ ) such that for $z \in B^{u}(y, 2 r)$

$$
C_{0}^{-1} \leq g(z) \leq C_{0} .
$$

Let $\eta^{u}(y)$ denote the connected component of $\xi^{u}(y) \cap B\left(x_{0}, r\right)$ which contains $y \in A$ and write $B^{u}=\bigcup_{y \in A} \eta^{u}(y)$. For any $y \in A$, by (b) we have that $l\left(f^{i}\left(\eta^{u}(y)\right)\right) \rightarrow \infty$ as $i \rightarrow \infty$, where $l(I)$ denotes the length of an interval $I$ in the unstable manifold. Since $f$ is topologically transitive, there exists $k_{1}>0$ such that $f^{k_{1}}\left(B^{u}\right)$ meets transversely one of the components of $f^{-1}(\mathscr{P}) \backslash \mathscr{P}$ along $E_{2}$. This intersection is denoted by $\mathscr{Q}^{(1)}$, and clearly we have $m_{y}^{u}\left(\mathscr{Q}^{(1)}\right)>0$ for $y \in \mathscr{Q}^{(1)}$. Furthermore we can show that $\mu\left(\mathscr{Q}^{(1)}\right)>0$. Indeed, since $\mu$ is $f$ invariant and is an SRB measure, 


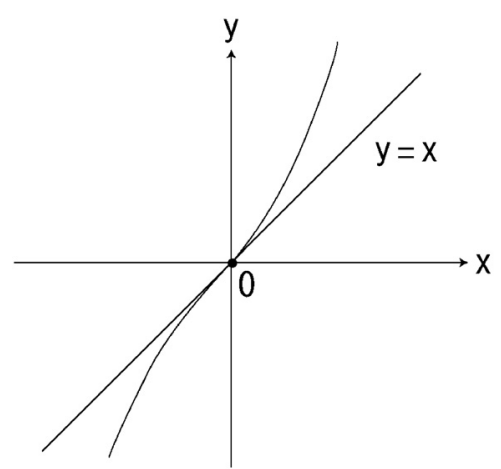

FIGURE 1. The graph of $\left.f\right|_{\tilde{W}_{\varepsilon_{1}}^{c u}(p)}$

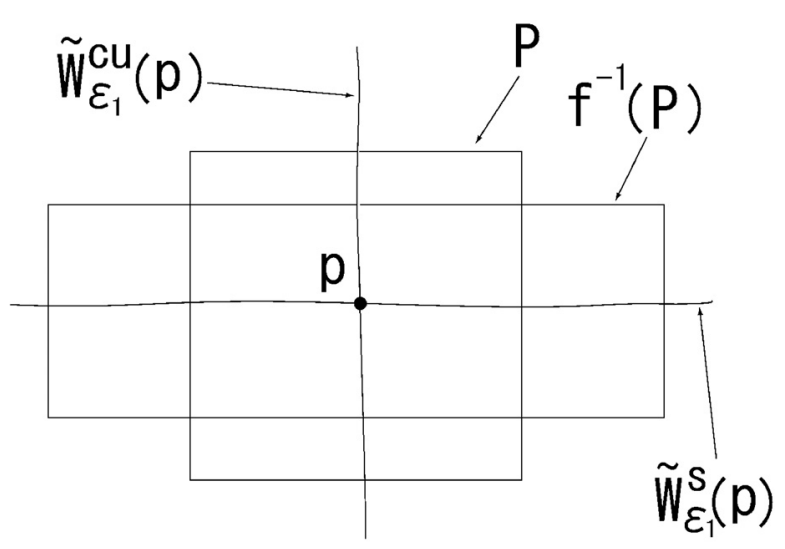

FIGURE 2. A transverse intersection of $f^{-1}(\mathscr{P}) \cap \mathscr{P}$

(3.4) $\quad \mu\left(\mathscr{Q}^{(1)}\right)=\mu\left(f^{-k_{1}}\left(\mathscr{Q}^{(1)}\right)\right)=\int \mu_{x}^{u}\left(f^{-k_{1}}\left(\mathscr{Q}^{(1)}\right)\right) d \mu(x)$

$$
\begin{aligned}
& =\int_{B^{u}}\left(\int_{f^{-k_{1}\left(\mathscr{Q}^{(1)}\right)}} g d m_{x}^{u}\right) d \mu(x) \\
& =\int_{B^{u}}\left(\int_{\mathscr{Q}^{(1)}} g\left(f^{-k_{1}}(z)\right)\left\|\left.D_{z} f^{-k_{1}}\right|_{E_{2}(z)}\right\| d m_{f^{k_{1}}(y)}^{u}(z)\right) d \mu(y) \\
& \geq C_{0}^{-1} \inf _{z \in \mathscr{Q}^{(1)}}\left\{\left\|\left.D_{z} f^{-k_{1}}\right|_{E_{2}(z)}\right\|\right\} \int_{B^{u}} m_{f^{k_{1}}(y)}^{u}\left(\mathscr{Q}^{(1)}\right) d \mu(y) \quad \\
& =C_{1} \int m_{x}^{u}\left(\mathscr{Q}^{(1)}\right) d \mu(x)
\end{aligned}
$$

where $C_{1}=C_{0}^{-1} \inf _{z \in \mathscr{Q}^{(1)}}\left\{\left\|\left.D_{z} f^{-k_{1}}\right|_{E_{2}(z)}\right\|\right\}$. The last term of (3.4) is positive because $\mu\left(B^{u}\right)>0$ and $m_{f^{k_{1}}(y)}^{u}\left(\mathscr{Q}^{(1)}\right)>0$ for $y \in B^{u}$. 
Define

$$
\mathscr{2}^{(i)}=\left\{z \in \mathscr{Q}^{(1)} \mid f^{j}(z) \in \mathscr{P},(1 \leq j \leq i)\right\} \quad(i \geq 2)
$$

and remark that $\mathscr{Q}^{(i)} \supset \mathscr{Q}^{(j)}, \quad f^{i}\left(\mathscr{Q}^{(i)}\right) \cap f^{j}\left(\mathscr{Q}^{(j)}\right)=\emptyset$ for $1 \leq i<j \quad$ and $\bigcup_{i \geq 1} f^{i}\left(\mathscr{Q}^{(i)}\right) \subset \mathscr{P}$. Let $\pi: \mathscr{Q}^{(1)} \rightarrow \tilde{W}_{\varepsilon}^{c u}(p)$ be the projection sliding along local stable manifolds. Since the fixed point $p$ satisfies the condition of Lemma 3.3, there exists $L_{1}>0$ such that

$$
m_{x}^{u}\left(\mathscr{Q}^{(i)}\right) \geq L_{1} m_{p}^{u}\left(\pi\left(\mathscr{Q}^{(i)}\right)\right)
$$

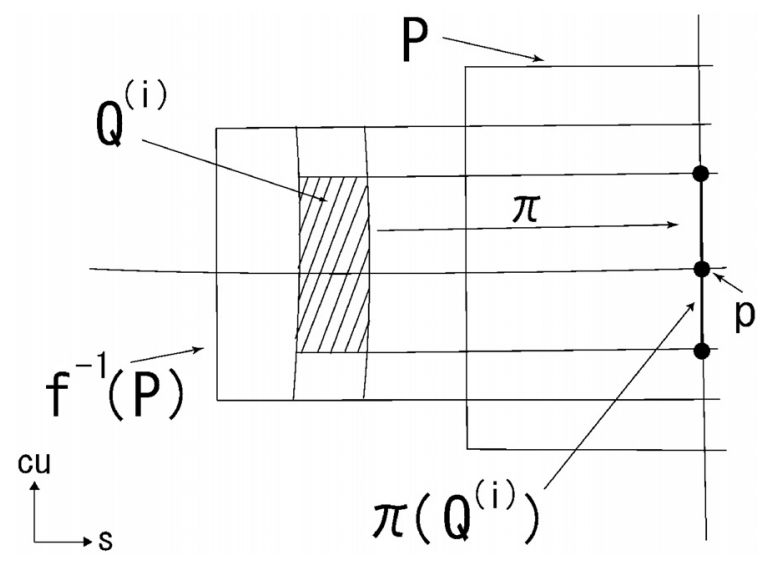

FIGURE 3. The figure of $2^{(i)}$

for any $x \in \mathscr{Q}^{(1)}$ (see Figure 3). In (3.4), replacing $\mathscr{2}^{(1)}$ by $\mathscr{Q}^{(i)}$, we have

$$
\mu\left(\mathscr{Q}^{(i)}\right) \geq C_{1} \int m_{x}^{u}\left(\mathscr{Q}^{(i)}\right) d \mu(x) \quad(i \geq 1) .
$$

By (3.5), (3.6) and $m_{p}^{u}\left(\pi\left(\mathscr{Q}^{(i)}\right)\right)=m_{p}^{u}\left(f^{-i}\left(\pi\left(\mathscr{Q}^{(1)}\right)\right)\right)$,

$$
\mu\left(\mathscr{Q}^{(i)}\right) \geq C_{1} L_{1} m_{p}^{u}\left(f^{-i}\left(\pi\left(\mathscr{Q}^{(1)}\right)\right)\right) .
$$

Then, since $\mu$ is $f$-invariant,

$$
\mu\left(\sum_{i=1}^{\infty} f^{i}\left(\mathscr{Q}^{(i)}\right)\right)=\sum_{i=1}^{\infty} \mu\left(f^{i}\left(\mathscr{Q}^{(i)}\right)\right)=\sum_{i=1}^{\infty} \mu\left(\mathscr{Q}^{(i)}\right) \geq C_{1} L_{1} \sum_{i=1}^{\infty} m_{p}^{u}\left(f^{-i}\left(\pi\left(\mathscr{Q}^{(1)}\right)\right)\right) .
$$

The last expression above goes to $\infty$ by Lemma 3.2. This is a contradiction with $\mu\left(\mathbf{T}^{2}\right)=1$. Therefore $\left\|\left.D_{p} f\right|_{E_{2}(p)}\right\|>1$, i.e., $p$ is hyperbolic. This completes the proof.

\section{Proof of Theorem B}

In this section we deal with a $C^{1+\alpha}$-diffeomorphism $f$ of $\mathbf{T}^{2}(0<\alpha<1)$ which has a non-hyperbolic fixed point $p$ and satisfies the following three assumptions: 
Assumption 1. There exist a norm $\|\cdot\|$ on $\mathbf{T}^{2}, 0<\lambda<1$ and a $D_{x} f$ invariant decomposition $\mathrm{T}_{x} \mathbf{T}^{2}=E_{1}(x) \oplus E_{2}(x)$ into subspaces $E_{1}(x)$ and $E_{2}(x)$ which satisfy

$$
\left\|\left.D_{x} f\right|_{E_{1}(x)}\right\| \leq \lambda, \quad\left\|\left.D_{x} f\right|_{E_{2}(x)}\right\| \begin{cases}=1 & (x=p), \\ >1 & (x \neq p) .\end{cases}
$$

For any $0<\varepsilon<1$, if we denote the local stable and unstable manifolds $\tilde{W}_{\varepsilon}^{s}(x)$ and $\tilde{W}_{\varepsilon}^{u}(x)$ at $x \in \mathbf{T}^{2}$ by

$$
\begin{aligned}
& \tilde{W}_{\varepsilon}^{s}(x)=\left\{y \in \mathbf{T}^{2} \mid d\left(f^{n}(y), f^{n}(x)\right) \leq \varepsilon,(n \geq 0)\right\} \quad \text { and } \\
& \tilde{W}_{\varepsilon}^{u}(x)=\left\{y \in \mathbf{T}^{2} \mid d\left(f^{-n}(y), f^{-n}(x)\right) \leq \varepsilon,(n \geq 0)\right\}
\end{aligned}
$$

respectively, then it follows from Assumption 1 that $\tilde{W}_{\varepsilon}^{s}(x)$ and $\tilde{W}_{\varepsilon}^{u}(x)$ are $C^{1+\alpha}$ manifolds with $T_{x} \tilde{W}_{\varepsilon}^{s}(x)=E_{1}(x)$ and $T_{x} \tilde{W}_{\varepsilon}^{u}(x)=E_{2}(x)$ ([9]). To obtain the Lipschitz continuity of the holonomy map along local stable manifolds (Lemma 4.3), we impose the following assumption.

Assumption 2. (1) For any $x \in \mathbf{T}^{2}$ and $0<\varepsilon<1$, each local unstable manifold $\tilde{W}_{\varepsilon}^{u}(x)$ is a $C^{2}$-embedding and (2) $W^{u}$-foliation $\left\{\tilde{W}_{\varepsilon}^{u}(x) \mid x \in \mathbf{T}^{2}\right\}$ is $C^{2}$-continuous, i.e. the correspondence $x \mapsto \tilde{W}_{\varepsilon}^{u}(x)$ is $C^{2}$-continuous.

Assumption 3. If we identify $\tilde{W}_{\varepsilon}^{u}(p)$ with $I_{\varepsilon}=[-\varepsilon, \varepsilon]$, then the graph of $\left.f\right|_{\tilde{W}_{\varepsilon}^{u}(p)}$ can be represented as

$$
\left.f\right|_{\tilde{W}_{\varepsilon}^{u}(p)}(x)= \begin{cases}x+x^{1+\alpha}+o\left(x^{2}\right) & (x \geq 0), \\ x-|x|^{1+\alpha}-o\left(x^{2}\right) & (x<0) .\end{cases}
$$

Assumption 3 implies that $f$ is of $C^{1+\alpha}$ on $\tilde{W}_{\varepsilon}^{u}(p)$ and it is crucial in proving the existence of an SRB measure.

Remark 4.1 ([19] Chapter VIII 8.8). We can construct a $C^{1+\alpha_{-}}$ diffeomorphism $f$ above as follows: Let $f_{0}$ be a hyperbolic toral automorphism of $\mathbf{T}^{2}$ with two different eigenvalues $0<\lambda_{1}<1<\lambda_{2}$. We slowly deform $f_{0}$ near the origin along only unstable direction until it satisfies Assumptions 1 and 3 .

By Assumption 1, $f$ is partially hyperbolic with contracting direction but not an Anosov diffeomorphism and there exists $\delta>0$ such that if $d(x, y)<\delta$, then $\tilde{W}_{\varepsilon}^{s}(x)$ and $\tilde{W}_{\varepsilon}^{u}(y)$ have a single transverse intersection point $[x, y]=$ $\tilde{W}_{\varepsilon}^{s}(x) \cap \tilde{W}_{\varepsilon}^{u}(y)$ ([9] Theorem 5.5). Thus $f$ is expansive, i.e. there exists $\eta>0$ such that if $x, y \in \mathbf{T}^{2}$ and $d\left(f^{i}(x), f^{i}(y)\right)<\eta(i \in \mathbf{Z})$ then $x=y$.

Moreover we can check that $f$ satisfies the uniformly shadowing property ([2] Theorem 5.4, [3] Theorem 2.2.17).

A sequence $\left\{x_{i}\right\}_{i \in \mathbf{Z}} \subset \mathbf{T}^{2}$ is called a $\beta$-pseudo orbit for $f$ if $d\left(f\left(x_{i}\right), x_{i+1}\right)<\beta$ for all $i \in \mathbf{Z}$. A point $x \in \mathbf{T}^{2}$ is called an $\alpha$-shadowing point for a $\beta$-pseudo orbit $\left\{x_{i}\right\}_{i \in \mathbf{Z}}$ if $d\left(f^{i}(x), x_{i}\right)<\alpha(i \in \mathbf{Z})$. We call that $f$ satisfies uniformly shadowing 
property if for any $\alpha>0$ there exists $\beta>0$ such that for a $\delta$-pseudo orbit there exists at least one $\alpha$-shadowing point. Since $f$ is expansive and satisfies the uniformly shadowing property, $f$ is topologically conjugate to some hyperbolic toral automorphism ([8] Theorem) and is topologically transitive.

To conclude Theorem $\mathrm{B}$ it is enough to show the following:

Proposition 4.2. If $0<\alpha<1$, then $f$ admits an $S R B$ measure.

In [11] $\mathrm{Hu}$ and Young gave a direct proof of the Lipschitz continuity of the holonomy map along the stable leaves for $C^{2}$-almost Anosov diffeomorphisms. We emphasize that in their proof they use only Assumptions 1 and 2. So the Lipschitz continuity also holds for our case:

Lemma 4.3 ([1] Proposition 2.5). There exists $L>0$ such that for any $y \in \mathbf{T}^{2}$, interval $J \subset \tilde{W}_{\varepsilon}^{u}(y)$ and $q \in \tilde{W}_{\varepsilon}^{s}(y)$ with $d(y, q)<\delta$,

$$
L^{-1} \ell(J) \leq \ell([J, q]) \leq L \ell(J) .
$$

By Assumption 3, we have the following ([24] p. 180):

LemMA 4.4 ([11], [24]). For any interval $J \subset \tilde{W}_{\varepsilon}^{u}(p)$

$$
\sum_{i=0}^{\infty} \ell\left(f^{-i}(J)\right)<\infty .
$$

For any $z \in \mathbf{T}^{2}$ we denote by

$$
\mathscr{R}_{\varepsilon^{\prime}}(z)=\left\{[y, x] \mid y \in \tilde{W}_{\varepsilon^{\prime}}^{u}(z), x \in \tilde{W}_{\varepsilon^{\prime}}^{s}(z)\right\} \quad\left(0<\varepsilon^{\prime} \leq \varepsilon\right)
$$

a rectangle of $z$. Combining Lemma 4.3 with the proofs of Proposition 3.1 in [11] and Lemma 5 in [24], we have the following:

LeMma 4.5 ([11], [24]). For any small rectangle $\mathscr{P}$ of $p$, there exist $\delta_{1}>0$ and $K>0$ such that for any $x \in \mathbf{T}^{2}$ and any interval $J \subset \tilde{W}_{\varepsilon}^{u}(x)$ with $l(J) \leq \delta_{1}$ and $J \cap \mathscr{P}=\emptyset$

$$
\frac{1}{K} \leq \frac{\left|\operatorname{det}\left(\left.D_{y} f^{-n}\right|_{E_{2}(y)}\right)\right|}{\left|\operatorname{det}\left(\left.D_{z} f^{-n}\right|_{E_{2}(z)}\right)\right|} \leq K \quad(y, z \in \gamma, n \geq 1)
$$

where $\operatorname{det}\left(\left.D_{y} f\right|_{E_{2}(y)}\right)$ denotes the Jacobian at $y$ of $f$ restricted to $E_{2}(y)$.

Now we introduce here the notion of a Markov partition. For any rectangle $\mathscr{R}$ and $x \in \mathscr{R}$, let $\gamma^{\sigma}(x)$ be the stable $(\sigma=s)$ and unstable $(\sigma=u)$ leaf which is the connected component of $\tilde{W}_{\varepsilon}^{\sigma}(x) \cap \mathscr{R}$ containing $x$. A rectangle $\mathscr{R} \subset \mathbf{T}^{2}$ is said to be proper if $\mathrm{Cl}(\operatorname{int}(\mathscr{R}))=\mathscr{R}$ where $\mathrm{Cl}(A)$ and $\operatorname{int}(A)$ denote the closure and interior of a set $A$ respectively. We say that $\left\{\mathscr{R}_{i}\right\}_{i=0}^{r-1}$ is a Markov partition if (i) each $\mathscr{R}_{i}$ is proper, (ii) $\left\{\mathscr{R}_{i}\right\}_{i=0}^{r-1}$ is a cover of $\mathbf{T}^{2}$, (iii) 
$\operatorname{int}\left(\mathscr{R}_{i}\right) \cap \operatorname{int}\left(\mathscr{R}_{j}\right)=\emptyset$ for $i \neq j$ and (iv) for any $x \in \operatorname{int}\left(\mathscr{R}_{i}\right) \cap f^{-1}\left(\operatorname{int}\left(\mathscr{R}_{j}\right)\right)$, $f\left(\gamma^{s}(x)\right) \subset \gamma^{s}(f(x))$ and $f\left(\gamma^{u}(x)\right) \supset \gamma^{u}(f(x))$.

Since $f$ is expansive and satisfies uniformly shadowing property, $f$ has a Markov partition $\left\{\mathscr{R}_{i}\right\}_{i=0}^{r-1}$ with arbitrary diameter (see [3]). So we can assume that the diameter of $\left\{\mathscr{R}_{i}\right\}_{i=0}^{r-1}$ is less than $0<\varepsilon_{2}<\delta / 2$. Moreover, since $f$ is topologically conjugate to some hyperbolic toral automorphism, each element of $\left\{\mathscr{R}_{i}\right\}_{i=0}^{r-1}$ is homeomorphic to a parallelogram ([22] Theorem 4.1) and its boundary consists of two stable leaves and two unstable leaves.

We consider elements of $\left\{\mathscr{R}_{i}\right\}_{i=0}^{r-1}$ containing the fixed point $p$. Then $p$ is contained in the interior of some $\mathscr{R}_{i}$ or, in the boundaries of some $\mathscr{R}_{i}$ s. If the latter happens, one of the boudary leaves of $\mathscr{R}_{i}$ is an unstable or stable leaf with p. This implies that the cardinarity of the set of all $\mathscr{R}_{i}$ containing $p$ is less than 4. Since we can assume that all the eigenvalues of $D_{p} f$ are positive (by replacing $f$ by $f^{2}$, if necessary), we have

(a) $f\left(\operatorname{int}\left(\mathscr{R}_{i}\right)\right) \cap \operatorname{int}\left(\mathscr{R}_{i}\right) \neq \emptyset$ whenever $p \in \mathscr{R}_{i}$, and

(b) $f\left(\operatorname{int}\left(\mathscr{R}_{i}\right)\right) \cap \operatorname{int}\left(\mathscr{R}_{j}\right)=\emptyset$ whenever $p \in \mathscr{R}_{i} \cap \mathscr{R}_{j}(i \neq j)$.

If we take a neighborhood $\mathscr{P}$ of $p$ where

$$
\mathscr{P}=\operatorname{int}\left(\bigcup_{p \in \mathscr{R}_{i}} \mathscr{R}_{i}\right),
$$

then $f$ is uniformly hyperbolic outside $\mathscr{P}$ (by Assumption 1). By (a) and (b) we have $f(\mathscr{P}) \cap \mathscr{P}=\operatorname{int}\left(\bigcup_{p \in \mathscr{R}_{i}} f\left(\mathscr{R}_{i}\right) \cap \mathscr{R}_{i}\right)$.

Let $R(x)$ be the smallest positive integer such that $\left(f^{R}\right)(x)=f^{R(x)}(x) \in$ $\mathbf{T}^{2} \backslash \mathscr{P}$ for $x \in \mathbf{T}^{2} \backslash \mathscr{P}$. By Assumption 1, the first return map $f^{R}$ is defined for $m$-a.e. $x \in \mathbf{T}^{2} \backslash \mathscr{P}$ where $m$ denotes the Lebesgue measure on $\mathbf{T}^{2}$. We set

$$
\Gamma_{i}=\left\{y \in \mathbf{T}^{2} \backslash \mathscr{P} \mid R(y)=i\right\} \quad(i \geq 1),
$$

then $f^{R}(x)=f^{i}(x)$ for $x \in \Gamma_{i}$. We define

$$
\mathscr{Q}^{(i)}=\left\{z \in f^{-1}(\mathscr{P}) \backslash \mathscr{P} \mid f^{j}(z) \in \mathscr{P},(1 \leq j \leq i)\right\} \quad(i \geq 1) .
$$

Then $f^{i}\left(\mathscr{Q}^{(i)}\right) \cap f^{j}\left(\mathscr{Q}^{(j)}\right)=\emptyset$ for $i \neq j, \mathscr{P}=\bigcup_{i \geq 1} f^{i}\left(\mathscr{Q}^{(i)}\right)$ and $\mathscr{Q}^{(i)}=\bigcup_{j \geq i+1} \Gamma_{j}$.

For any rectangle $\mathscr{R}$, any unstable leaf $\gamma^{u}$ and any $\rho>0$, we say that $\mathscr{V}_{\rho} \subset \mathscr{R}$ is a $u$-subset of $\gamma^{u}$ with radius $\rho$ if $\mathscr{V}_{\rho}=\bigcup_{y \in \tilde{B}^{s}(x, \rho)} \gamma^{u}(y)$ for $x \in \gamma^{u}$, where $\tilde{B}^{s}(x, \rho)$ denotes the closed ball in $\tilde{W}_{\varepsilon}^{s}(x)$ centered at $x$ with radius $\rho$. For any interval $\omega \subset \gamma^{u}, \mathscr{S}_{\omega}$ is an s-subset corresponding to $\omega$ if $\mathscr{S}_{\omega}=\bigcup_{y \in \omega} \gamma^{s}(y)$. We denote $\partial^{s}(\mathscr{R})$ the two stable leaves which contain the different extreme points of any unstable leaf $\gamma^{u} \subset \mathscr{R}$. $\partial^{u}(\mathscr{R})$ is also defined. The boudary of $\mathscr{R}, \partial(\mathscr{R})$, is represented as $\partial(\mathscr{R})=\partial^{s}(\mathscr{R}) \cup \partial^{u}(\mathscr{R})$.

Let $\xi^{u}$ be the measurable partition subordinate to $W^{u}$-foliation. For an $f^{R}$-invariant probability measure $v$ on $\mathbf{T}^{2} \backslash \mathscr{P}$, let $\left\{v_{x}^{u}\right\}(v$-a.e. $x)$ denote the canonical system of conditional measures of $v$ w.r.t. $\xi^{u}$ and $m_{x}^{u}$ denote the Lebesgue measure on $\gamma^{u}(x)$. 
Lemma 4.6. There exists an $f^{R}$-invariant Borel probability measure $\mu$ such that $\mu_{x}^{u} \ll m_{x}^{u}(\mu-a . e . x)$.

Proof. Let $\gamma_{0}$ be an unstable leaf which intersects one of the components of $f^{-1}(\mathscr{P}) \backslash \mathscr{P}$ and $m_{0}^{u}$ be the Lebesgue measure on $\gamma_{0}$. To simplicity we assume that $m_{0}^{u}\left(\gamma_{0}\right)=1$, and so define a probability measure of $\mathbf{T}^{2} \backslash \mathscr{P}$ by

$$
\mu_{n}=\frac{1}{n} \sum_{j=0}^{n-1} \sum_{i=1}^{\infty}\left(f^{R}\right)_{*}^{j}\left(\left.m_{0}^{u}\right|_{\Gamma_{i}}\right) \quad(n \geq 1) .
$$

Here $\left(f^{R}\right)_{*}^{j}\left(\left.m_{0}^{u}\right|_{\Gamma_{i}}\right)$ is the push-forward of $\left.m_{0}^{u}\right|_{\Gamma_{i}}$ by $\left(f^{R}\right)^{j}$. Then there exist a probability measure $\mu$ on $\mathbf{T}^{2} \backslash \mathscr{P}$ and a subsequence $\left\{\mu_{n_{j}}\right\}_{j \geq 1} \subset\left\{\mu_{n}\right\}_{n \geq 1}$ such that $\mu_{n_{j}} \rightarrow \mu(j \rightarrow \infty)$. Clearly $\mu$ is $f^{R}$-invariant.

To obtain the conclusion it is enough to show that there exists $K_{1}>0$ such that for $\mu$-a.e. $x$ and any interval $\omega \subset \gamma^{u}(x)$

$$
\frac{1}{K_{1}} m_{x}^{u}(\omega) \leq \mu_{x}^{u}(\omega) \leq K_{1} m_{x}^{u}(\omega) .
$$

For any $x \in \mathbf{T}^{2}$, to simplicity set $\gamma=\gamma^{u}(x)$ and choose any interval $\omega \subset \gamma$. $\mathscr{S}_{\omega}$ denotes the s-subset corresponding to $\omega$ and $\mathscr{V}_{\rho}$ the u-subset of $\gamma$ with radius $0<\rho<\delta / 2$. Firstly we prove that there exists $K_{0}>0$ such that

$$
\frac{1}{K_{0}} \frac{m_{x}^{u}(\omega)}{m_{x}^{u}(\gamma)} \leq \frac{\mu_{n}\left(\mathscr{V}_{\rho} \cap \mathscr{S}_{\omega}\right)}{\mu_{n}\left(\mathscr{V}_{\rho}\right)} \leq K_{0} \frac{m_{x}^{u}(\omega)}{m_{x}^{u}(\gamma)} .
$$

To see this, we use the arguments in the proof of Lemma 5.2 in [11] and Theorem 1 in [25]. We set $\gamma_{i}^{j}=\left(f^{R}\right)^{j}\left(\gamma_{0} \cap \Gamma_{i}\right) \cap \mathscr{V}_{\rho}$. Since $f$ is topologically transitive, for $n>0$ large enough there exist $i \geq 1$ and $0 \leq j \leq n-1$ such that $\gamma_{i}^{j} \neq \emptyset$. By Lemma 4.3 there exists $L>0$ such that for $0 \leq j \leq n-1$ and $i \geq 1$ with $\gamma_{i}^{j} \neq \emptyset$,

$$
\frac{1}{L^{2}} \frac{m_{x}^{u}(\omega)}{m_{x}^{u}(\gamma)} \leq \frac{m_{\gamma_{i}^{j}}^{u}\left(\gamma_{i}^{j} \cap S_{\omega}\right)}{m_{\gamma_{i}^{j}}^{u}\left(\gamma_{i}^{j}\right)} \leq L^{2} \frac{m_{x}^{u}(\omega)}{m_{x}^{u}(\gamma)}
$$

By Lemma 4.5, (4.3) and by using the fact that $\left(\sum_{i=1}^{\infty} a_{i}\right) /\left(\sum_{i=1}^{\infty} b_{i}\right) \leq$ $\sup _{1 \leq i}\left\{a_{i} / b_{i}\right\}$ for $a_{i}, b_{i}>0(i \geq 1)$ and $\sum_{i=1}^{\infty} a_{i}<\infty, \sum_{i=1}^{\infty} b_{i}<\infty$, we can find $K>0$ such that

$$
\frac{1}{K L^{2}} \frac{m_{x}^{u}(\omega)}{m_{x}^{u}(\gamma)} \leq \frac{\left(f^{R}\right)_{*}^{j}\left(m_{0}^{u}\right)\left(\mathscr{V}_{\rho} \cap \mathscr{S}_{\omega}\right)}{\left(f^{R}\right)_{*}^{j}\left(m_{0}^{u}\right)\left(\mathscr{V}_{\rho}\right)} \leq K L^{2} \frac{m_{x}^{u}(\omega)}{m_{x}^{u}(\gamma)} .
$$

By (4.4) and the fact above again, we have (4.2).

We can choose $\mathscr{V}_{\rho}$ such that their boundary $\partial\left(\mathscr{V}_{\rho}\right)$ has $\mu$-zero measure. Indeed, it is enough to show that $\mu\left(\gamma^{s}\right)=0$ for any unstable leaf $\gamma^{s}$. Since there exist at most countable $\rho>0$ such that $\mu\left(\partial^{u}\left(\mathscr{V}_{\rho}\right)\right)>0$, except for such $\rho>0$, we have that $\mu\left(\partial^{u}\left(\mathscr{V}_{\rho}\right)\right)=0$. If we have the claim above, then $\mu\left(\partial^{s}\left(\mathscr{V}_{\rho}\right)\right)=0$. Therefore $\mu\left(\partial\left(\mathscr{V}_{\rho}\right)\right)=0$.

For any stable leaf $\gamma^{s}$ and $0<\eta<\varepsilon_{2}$ we set 


$$
U\left(\gamma^{s}, \eta\right)=\left\{[y, z] \in \mathbf{T}^{2} \backslash \mathscr{P} \mid z \in \gamma^{s}, d(z, y)<\eta\right\} .
$$

Then

$$
U\left(\gamma^{s}, \eta\right)=\bigcup_{k=1}^{l}\left(U\left(\gamma^{s}, \eta\right) \cap \mathscr{R}_{m_{k}}\right)
$$

for $\quad \mathscr{R}_{m_{k}} \neq \mathscr{R}_{0}(k=1, \ldots, l)$ with $U\left(\gamma^{s}, \eta\right) \cap \mathscr{R}_{m_{k}} \neq \emptyset$. For any $k=1, \ldots, l$, there exist $y_{k} \in \mathscr{R}_{m_{k}}$, an interval $\omega_{\eta}\left(y_{k}\right) \subset \gamma^{u}\left(y_{k}\right)$ and $\rho\left(y_{k}\right)>0$ such that (a) the $u$-subset $\mathscr{V}_{\rho\left(y_{k}\right)}$ of $\gamma^{u}\left(y_{k}\right)$ and the $s$-subset $\mathscr{S}_{\omega_{\eta}\left(y_{k}\right)}$ corresponding to $\omega_{\eta}\left(y_{k}\right)$ satisfy $U\left(\gamma^{s}, \eta\right) \cap \mathscr{R}_{m_{k}}=\mathscr{V}_{\rho\left(y_{k}\right)} \cap \mathscr{S}_{\omega_{\eta}\left(y_{k}\right)}$, (b) $m_{y_{k}}^{u}\left(\omega_{\eta}\left(y_{k}\right)\right) \rightarrow 0$ as $\eta \rightarrow 0$. By (4.2), for any $n \geq 1$,

$$
\mu_{n}\left(U\left(\gamma^{s}, \eta\right) \cap \mathscr{R}_{m_{k}}\right) \leq K_{0} \frac{m_{y_{k}}^{u}\left(\omega_{\eta}\left(y_{k}\right)\right)}{m_{y_{k}}^{u}\left(\gamma^{u}\left(y_{k}\right)\right)} \rightarrow 0 \quad(\eta \rightarrow 0) .
$$

Since $\bigcup_{k=1}^{l} U\left(\gamma^{s}, \eta\right) \cap \mathscr{R}_{m_{k}}$ contains $\gamma^{s}$ and is open in $\mathbf{T}^{2} \backslash \mathscr{P}$ w.r.t. the relative topology, we have

$$
\begin{aligned}
\mu\left(\gamma^{s}\right) & \leq \mu\left(\bigcup_{k=1}^{l} U\left(\gamma^{s}, \eta\right) \cap \mathscr{R}_{m_{k}}\right) \\
& \leq \limsup _{j \rightarrow \infty} \mu_{n_{j}}\left(\bigcup_{k=1}^{l} U\left(\gamma^{s}, \eta\right) \cap \mathscr{R}_{m_{k}}\right) .
\end{aligned}
$$

By (4.5) and (4.6), we have $\mu\left(\gamma^{s}\right)=0$.

Thus we can choose the finite measurable partition $\xi_{1}$ which consists of $\mathscr{V}_{\rho} \cap \mathscr{S}_{\omega_{j}}$ with $\mu\left(\partial\left(\mathscr{V}_{\rho} \cap \mathscr{S}_{\omega_{j}}\right)\right)=0 \quad(1 \leq i \leq q)$ and set $\xi^{u}=\left\{\gamma^{u}(x) \cap \mathscr{V}_{\rho} \cap \mathscr{S}_{\omega_{j}} \mid\right.$ $\left.x \in \mathscr{R}_{i}, \operatorname{int}\left(\mathscr{R}_{i}\right) \cap \mathscr{P}=\emptyset, \mathscr{V}_{\rho} \cap \mathscr{S}_{\omega_{j}} \in \xi_{1}\right\}$. Then we can find the sequence $\left\{\xi_{\ell}\right\}_{\ell \geq 1}$ of finite measurable partition such that

$$
\xi_{1} \leq \xi_{2} \leq \cdots \leq \bigvee_{\ell \geq 1} \xi_{\ell}=\xi^{u}
$$

By Doob's theorem we have $\mu_{x}^{\ell} \rightarrow \mu_{x}^{u}(\mu$-a.e. $x)$ as $\ell \rightarrow \infty$, where $\left\{\mu_{x}^{\ell}\right\}$ ( $\mu$-a.e. $x$ ) denotes the canonical system of conditional measures w.r.t. $\xi_{\ell}$. Here we remark that $\mu_{x}^{\ell}(A)=\mu\left(\mathscr{V}_{\rho_{\ell}} \cap \mathscr{S}_{\omega_{j}} \cap A\right) / \mu\left(\mathscr{V}_{\rho_{\ell}} \cap \mathscr{S}_{\omega_{j}}\right)$ for any $\mathscr{V}_{\rho_{\ell}} \cap \mathscr{S}_{\omega_{j}} \in \xi_{\ell}$, any Borel set $A$ and $x \in \mathscr{V}_{\rho_{i}} \cap \mathscr{S}_{\omega_{j}} \cap A$.

Since (4.2) holds for $\mathscr{V}_{\rho_{\ell}} \cap \mathscr{S}_{\omega_{j}} \in \xi_{l}(1 \leq j \leq q)$ instead of $\mathscr{V}_{\rho}$ and any interval $\omega \subset \omega_{j}$ with $\mu\left(\partial\left(\mathscr{V}_{\rho_{\ell}} \cap \mathscr{S}_{\omega}\right)=0\right.$, by taking $n \rightarrow \infty$ in (4.2), we have

$$
\frac{1}{K_{0}} \frac{m_{x}^{u}(\omega)}{m_{x}^{u}\left(\gamma \cap \mathscr{S}_{\omega_{j}}\right)} \leq \frac{\mu\left(\left(\mathscr{V}_{\rho_{\ell}} \cap \mathscr{S}_{\omega_{j}}\right) \cap \mathscr{S}_{\omega}\right)}{\mu\left(\mathscr{V}_{\rho_{\ell}} \cap \mathscr{S}_{\omega_{j}}\right)} \leq K_{0} \frac{m_{x}^{u}(\omega)}{m_{x}^{u}\left(\gamma \cap \mathscr{S}_{\omega_{j}}\right)} .
$$

Doob's theorem ensures that we have

$$
\frac{1}{K_{0}} \frac{m_{x}^{u}(\omega)}{m_{x}^{u}\left(\gamma^{u}(x) \cap \mathscr{S}_{\omega_{j}}\right)} \leq \mu_{x}^{u}(\omega) \leq K_{0} \frac{m_{x}^{u}(\omega)}{m_{x}^{u}\left(\gamma^{u}(x) \cap \mathscr{S}_{\omega_{j}}\right)} \quad(\mu \text {-a.e. } x)
$$


as $\ell \rightarrow \infty$ in (4.7). Since $\mathscr{R}_{i} \cap \mathscr{S}_{\omega_{j}}$ is a rectangle $(0 \leq i \leq r-1,1 \leq j \leq q)$, by Lemma 4.3 there exists $K_{1}>0$ such that for any $x \in \mathscr{R}_{i}$,

$$
\frac{1}{K_{1}}<m_{x}^{u}\left(\gamma^{u}(x) \cap \mathscr{S}_{\omega_{j}}\right)<K_{1}
$$

Therefore we have (4.1) by (4.8) and (4.9).

Proof of Proposition 4.2. Let $\mu$ be the $f^{R}$-invariant Borel probability measure on $\mathbf{T}^{2} \backslash \mathscr{P}$ in Lemma 4.6. Then

$$
\bar{\mu}=\mu+\sum_{i=1}^{\infty} f_{*}^{i}\left(\left.\mu\right|_{\mathscr{Q}^{(i)}}\right)
$$

is a finite measure. To see this, it is enough to prove that $\bar{\mu}(\mathscr{P})<\infty$.

Let $\pi: \mathscr{Q}^{(1)} \rightarrow \gamma^{u}(p)$ be the projection sliding along local stable manifolds. As in proof of Theorem A, by Lemma 4.3 there exists $L>0$ such that for any $x \in f^{-1}(\mathscr{P}) \backslash \mathscr{P}$,

$$
m_{x}^{u}\left(\mathscr{Q}^{(i)}\right) \leq \operatorname{Lm}_{p}^{u}\left(\pi\left(\mathscr{Q}^{(i)}\right)\right) \quad(i \geq 1) .
$$

By (4.1) there exists $K>0$ such that

$$
\mu\left(\mathscr{Q}^{(i)}\right)=\int \mu_{x}^{u}\left(\mathscr{Q}^{(i)}\right) d \mu(x) \leq K \int m_{x}^{u}\left(\mathscr{Q}^{(i)}\right) d \mu(x) \quad(i \geq 1) .
$$

By (4.11), (4.10) and $m_{p}^{u}\left(\pi\left(\mathscr{Q}^{(i)}\right)\right)=m_{p}^{u}\left(f^{-i}\left(\pi\left(\mathscr{Q}^{(1)}\right)\right)\right)$,

$$
\mu\left(\mathscr{Q}^{(i)}\right) \leq 2 K L \cdot m_{p}^{u}\left(f^{-i}\left(\pi\left(\mathscr{Q}^{(1)}\right)\right)\right) \quad(i \geq 1) .
$$

By $\mathscr{P}=\bigcup_{i=1}^{\infty} f^{i}\left(\mathscr{Q}^{(i)}\right), \quad f^{i}\left(\mathscr{Q}^{(i)}\right) \cap f^{j}\left(\mathscr{Q}^{(j)}\right)=\emptyset(i \neq j), \quad \bar{\mu}\left(f^{i}\left(\mathscr{Q}^{(i)}\right)\right)=\mu\left(\mathscr{Q}^{(i)}\right)$ and (4.12), we have

$$
\bar{\mu}(\mathscr{P})=\sum_{i=1}^{\infty} \mu\left(\mathscr{Q}^{(i)}\right) \leq 2 K L \sum_{i=1}^{\infty} m_{p}^{u}\left(f^{-i}\left(\pi\left(\mathscr{Q}^{(1)}\right)\right)\right) .
$$

Lemma 4.4 ensures that the last term above converges.

By Lemma 4.6 the normalized measure of $\bar{\mu}$ is an SRB measure. This concludes the proposition.

\section{REFERENCES}

[ 1 ] N. AokI, The series of nonlinear analysis (in Japanese) I, II, III and IV, Kyoritu Publ., 2004.

[2] N. AokI, Topological dynamics, Topics in general topology, North-Holland Math. Library 41, North-Holland, Amsterdam, 1989, 625-740.

[ 3 ] N. AoKi AND K. Hiraide, Topological theory of dynamical systems: recent advances, NorthHolland Math. Library 52, Elsevier North-Holland, Amsterdam, 1994.

[4] R. BowEN, Equilibrium states and the ergodic theory of anosov diffeomorphisms, Lecture notes in mathematics 470, Springer-Verlag, 1975, 67-76. 
[5] E. Catsigeras and H. EnRich, SRB measures of certain almost hyperbolic diffeomorphisms with a tangency, Discr. and Conti. Dynam. Syst. 7 (2001), 188-202.

[6] J. Franks, Anosov diffeomorphisms, Global analysis, Proc. Sympos. Pure Math. 14 (1970), 61-93.

[7] J. НАтомото, Ergodic measures of SRB attractors, 2004, preprint.

[8] K. Hiraide, Expansive homeomorphisms with the pseudo-orbit tracing property on compact surfaces, J. Math. Soc. Japan 40 (1994), 123-137.

[9] M. Hirsch, C. Pugh ANd M. Shub, Invariant manifolds, Lecture notes in math. 583, 1977.

[10] H. Hu, Conditions for the existence of SBR measures for "almost Anosov" diffeomorphisms, Discr. and Conti. Dynam. Syst. 5 (1999), 2331-2367.

[11] H. Hu AND L.-S. Young, Nonexistence of SBR measures for some diffeomorphism that are 'Almost Anosov', Ergod. Th. and Dynam. Sys. 15 (1995), 67-76.

[12] A. KatoK, Lyapunov exponents entropy and periodic orbits for diffeomorphisms, Inst. Hautes Études Sci. Publ. Math. 51 (1980), 137-173.

[13] F. Ledrappier and J. Strelcyn, A proof of the estimation from below in Pesin's entropy formula, Ergod. Th. and Dynam. Sys. 2 (1982), 203-219.

[14] F. Ledrappier AND L.-S. Young, The metric entropy of diffeomorphisms I and II, Ann. of Math. 122 (1985), 509-574.

[15] Y. I. Oseledec, A multiplicative ergodic theorem, Lyapunov characteristic numbers for dynamical systems, Trudy Moskov Mat. Ostc. 19 (1968), 179-210.

[16] Y. B. Pesin, Characteristic Lyapunov exponents and smooth ergodic theory, Math. Surveys 32 (1977), 55-114.

[17] Y. B. Pesin And Ya. SinaI, Gibbs measures for partially hyperbolic attractors, Ergod. Th. and Dynam. Sys. 2 (1982), 417-438.

[18] E. Pujals and M. Sambarino, Homoclinic tangencies and hyperbolicity for surface diffeomorphisms, Ann. Math. 151 (2000), 961-1023.

[19] C. Robinson, Dynamical systems: Stability, symbolic dynamics, and chaos, 2nd ed., Stu. in Adv. Math., CRC Press, 1999.

[20] V. A. RoHLIn, On the fundamental ideas of measure theory, Mat. Sbornik 25 (1949).

[21] D. Ruelle, A measure associated with axiom $A$ attractors, Amer. J. Math. 98 (1976), 619-654.

[22] E. RykKen, Markov partitions for hyperbolic toral automorphisms $\mathbf{T}^{2}$, Rocky Mountain $\mathbf{J}$. Math. 28 (1998), 1103-1124.

[23] YA. G. SinAI, Gibbs measures in ergodic theory, Uspehi Mat. Nauk 27 (1972), 21-64.

[24] L.-S. Young, Recurrence times and rates of mixing, Israel. J. Math. 110 (1999), 153-188.

[25] L.-S. Young, Statistical properties of dynamical systems with some hyperbolicity, Ann. Math. 147 (1998), 585-650.

\author{
Department of Mathematics \\ Tokyo Institute of Technology \\ O-OKayama, MegurO-KU \\ TOKYO, 152-8551 \\ JAPAN \\ E-mail: hatoj@math.titech.ac.jp
}

